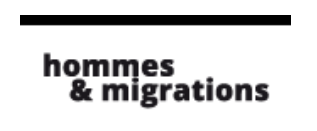

Hommes \& migrations

Revue française de référence sur les dynamiques

migratoires

$1317-1318 \mid 2017$

L'Europe en mouvement

\title{
Féminisation de la migration qualifiée : les raisons d'une invisibilité
}

\section{Speranta Dumitru}

\section{(2) OpenEdition \\ Journals}

Édition électronique

URL : http://journals.openedition.org/hommesmigrations/3914

DOI : 10.4000/hommesmigrations.3914

ISSN : 2262-3353

Éditeur

Musée national de l'histoire de l'immigration

Édition imprimée

Date de publication : 1 avril 2017

Pagination : 146-153

ISBN : 978-2-919040-38-4

ISSN : 1142-852X

Référence électronique

Speranta Dumitru, «Féminisation de la migration qualifiée : les raisons d'une invisibilité », Hommes \& migrations [En ligne], 1317-1318 | 2017, mis en ligne le 01 avril 2017, consulté le 15 septembre 2020. URL : http://journals.openedition.org/hommesmigrations/3914 


\section{FÉMINISATION DE LA MIGRATION QUALIFIÉE : LES RAISONS D'UNE INVISIBILITÉ}

Par SPERANTA DIMITRU, Maîtresse de Conférences en Sciences Politiques, Faculté de Droit, Université Paris-Descartes, laboratoire CERLIS

$\mathrm{D}$ ans la recherche sur les femmes migrantes, il existe une asymétrie entre le volume d'études dédiées à la « féminisation de la migration » et l'absence presque totale d'études évoquant la « féminisation de la migration qualifiée ". Cette asymétrie est surprenante à la lumière des données statistiques car, depuis longtemps, elles infirment l'existence d'un phénomène de féminisation de la migration en général, mais confirment la tendance à la féminisation de la migration qualifiée. Ainsi, en 2010, les femmes constituaient la majorité des migrants qualifiés présents dans 20 pays membres de l'Organisation de coopération et de développement économiques (OCDE), leur proportion augmentant de $45 \%$ en 1980 à $51 \%$ en 2010'. Cette tendance à la féminisation de la migration qualifiée est systématiquement observée depuis une décennie, lorsque les premières statistiques migratoires désagrégées par sexe et par niveau d'éducation sont apparues². Bien que les études sur les femmes migrantes qualifiées ne manquent plus aujourd'huỉ, l'expression "féminisation de la migration qualifiée » est rarement employée dans la littérature ${ }^{4}$. Comment expliquer cette absence ? L'absence d'analyses de la "féminisation de la migration qualifiée " contraste avec la fréquence de l'expression " féminisation de la migration " (tout court) dans les recherches sur les femmes migrantes. Cette dernière figure désormais comme entrée dans des encyclopédies et des dictionnaires prestigieux ${ }^{5}$, mais aucune référence aux femmes qualifiées n'y est faite. Bien au contraire, l'expression « féminisation de la migration » est systématiquement associée aux emplois peu qualifiés. Par exemple, selon l'une des définitions, "la féminisation de la migration produit des formes genrées

\footnotetext{
1. Speranta Dumitru, Abdeslam Marfouk 2. La première base de données a été construite en 2007 par Jean-Christophe Dumont, John P. Martin, Gilles Spielvogel, Women on the Move: The Neglected Gender Dimension of the Brain Drain, IZA Discussion Paper n² 2920, 2007. Elle a été suivie par Frédéric Docquier, B. Lindsay Lowell, Abdeslam Marfouk « A gendered assessment of highly-skilled emigration ", in Population and Development Review, vol. 35, n² 2, 2008, pp. $297-321$. 3. Voir le diagnostic d'Eleonore Kofman, "The invisibility of skilled female migrants and gender relations in studies of skilled migration in Europe ", in International Journal of Population Geography, vol. 6, 2000, pp. 45-59; Christine Catarino et Mirjana Morokvasic, «Femmes, genre, migration et mobilités », in Revue européenne des migrations internationales, vol. 21, $n^{\circ}$ 1, 2005, pp. 7-27.4. Voir, par exemple, Parvati Raghuram, "Gendering skilled migratory streams: Implications for conceptualizations of migration ", in Asian and Pacific Migration Journal, $n^{\circ}$ 4, 2009, pp. 429-457; Jean-Baptiste Meyer, Fan Wang Miao, Yue Zhao, "Visualizing the diaspora: new options ", in Abel Chikanda, Jonathan Crush, Margaret Walton-Roberts (dir.), Diasporas, Development and Governance, Berlin, Springer, 2016, pp. 205-220. 5. Teal K. Rothschild, "Feminization of migration ", in Jodi O'Brien (dir.), Encyclopedia of Gender and Society, Thousand Oaks, Sage Publications, 2009, pp. 321-325; Mako Yoshimura, "Feminization of labor migration ", in George Ritzer (dir.), Blackwell Encyclopedia of Sociology, Oxford, Blackwell Publishing, 2007, pp. 1715-1717; Donna R Gabaccia, « Feminization of migration », in Nancy A. Naples (dir.), The Wiley Blackwell Encyclopedia of Gender and Sexuality Studies, New York, John Wiley \& Sons, 2016.
} 
de migration, en particulier en termes de travailleuses domestiques ou de care fournies à domicile ou dans les maisons de santé; le trafic des femmes pour l'industrie du sexe et la migration organisée pour le mariage ${ }^{6} »$. Comment expliquer ce contraste?

Cet article explique la fréquence de l'expression "féminisation de la migration " dans la littérature, ainsi que son association aux emplois peu qualifiés comme une façon d'étayer la théorie de la division internationale du travail. Cette théorie, qui émerge à la fin des années 1970, ne porte pas sur la migration en particulier, mais sur la mondialisation plus généralement. Elle soutient que la mobilité du capital produit, entre autres conséquences, une féminisation de l'emploi dans les pays en développement et que celle-ci pousse ces femmes à migrer vers les pays développés. Dans les recherches inspirées par cette théorie, la « féminisation de la migration » est alors analysée comme illustrant la division internationale du travail, les études se concentrant sur les femmes originaires des pays en développement travaillant dans les emplois peu qualifiés, notamment dans le secteur tertiaire. Le phénomène de la féminisation de la migration qualifiée est ainsi obscurci au profit d'une théorie compréhensive de la mondialisation.

\section{La féminisation de la migration internationale en question}

Il est difficile de comprendre le succès de l'expression "féminisation de la migration internationale " compte tenu des statistiques migratoires disponibles. Dans la littérature sur les femmes migrantes, cette formule est employée avec l'une des trois significations suivantes: le nombre absolu des femmes migrantes a aug- menté ; les femmes migrantes représenteraient la majorité des migrants internationaux; les femmes représenteraient une proportion croissante dans la migration internationale. Or, pour chacune de ces significations - croissance en nombre, en proportion, majorité -, les statistiques migratoires suggèrent qu'il serait plus judicieux de parler de " masculinisation " plutôt que de "féminisation " de la migration, si l'un de ces termes devait être utilisé.

Premièrement, si par « féminisation » on entend une augmentation du nombre de femmes migrantes, les données de la Division population des Nations unies montrent que ce nombre a augmenté de 36 à 117 millions entre 1960 et 2015 (voir tableau 1), mais que le nombre d'hommes migrants a, lui aussi, augmenté de 40 à 126 millions pendant cette même période. De plus, la progression du nombre des migrants, notamment des femmes, est en partie fictive si l'on observe qu'une augmentation importante a lieu en 1990, date qui coïncide avec l'éclatement de l'Union soviétique en 15 pays indépendants. Pour les statistiques migratoires, ce changement géopolitique a eu pour effet de transformer le statut de personnes considérées comme des migrants internes en migrants internationaux, sans que ces personnes aient traversé de frontière. En 1990, dans l'ensemble des pays qui faisaient autrefois partie de I'URSS, on dénombrait un total de près de 30 millions de migrants internationaux, contre 3,3 millions en 1985. La désagrégation de l'Union soviétique a ajouté au nombre total de migrants internationaux, environ 26,7 millions de personnes, dont 15,3 millions de femmes migrantes? Deuxièmement, si par "féminisation de la migration " on entend dire que les femmes auraient dépassé le seuil de $50 \%$ de la migration internationale, les données des Nations unies ne permettent pas de conjecturer l'existence 
REPÉRAGE

\section{Tableau 1. Effectif des migrants internationaux, femmes et hommes, en milliers, 1960-2015}

\begin{tabular}{llllllll}
\hline & 1960 & 1970 & 1980 & 1990 & 2000 & 2010 & 2015 \\
\hline Total (femmes et hommes) & 77115 & 84460 & 101983 & 152563 & 172703 & 221714 & 243700 \\
\hline Hommes & 40869 & 44256 & 53302 & 77747 & 87885 & 114614 & 126115 \\
\hline Femmes & 36246 & 40204 & 48681 & 74816 & 84818 & 107100 & 117585 \\
\hline$\%$ femmes & 47 & 47,6 & 47,7 & 49 & 49,1 & 48,3 & 48,2 \\
\hline
\end{tabular}

Source : Division de la population des Nations unies (révisions 2008 et 2015) et calculs de l'auteure.

d'un tel phénomène. Durant un demi-siècle, les hommes restent majoritaires, mais on a rarement évoqué la « masculinisation » de la migration internationale ${ }^{8}$.

Enfin, si par « féminisation » on entend une augmentation de la proportion des femmes dans la migration internationale, on observe que cette augmentation a été très faible au cours d'un demisiècle, passant de $47 \%$ en 1960 à $48,2 \%$ en 2015 . De plus, il serait difficile de parler de féminisation comme d'une tendance nouvelle car les données de stock ne permettent pas d'identifier si ce sont les flux d'entrée, de sortie ou la mortalité des migrants qui l'expliquent. Comme l'espérance de vie des femmes est plus élevée, les statistiques basées sur les stocks font apparaître une " féminisation » de la migration beaucoup plus importante que ne le montrent les données de flux d'entrée dans les principaux pays d'immigration.

Il est d'autant plus difficile de comprendre le succès de la formule « féminisation de la migration ", d'autant qu'elle s'est imposée après et en dépit de la publication des premières statistiques des migrations internationales. Dès 1990,
Hania Zlotnik, directrice de la Division population des Nations unies, notait que la proportion des femmes représentait $47 \%$ de la migration internationale en 1970-19809. La publication, en 1998, des premières estimations des stocks des migrants internationaux par les Nations unies montrait que la proportion des femmes restait stable depuis les années 1960.

L'usage de l'expression "féminisation de la migration internationale " non seulement n'a pas tari après la publication des données statistiques, mais s'est amplifié au cours des années 2000. Un nombre croissant d'études sur les femmes migrantes utilisent cette expression dans le titre : " La féminisation de la migration de travail comme violence contre les femmes ${ }^{10}$ ", " La féminisation de la migration : le care et le nouvel impérialisme émotionnel" ", "La féminisation de la migration et le trafic des femmes ${ }^{12}$ ", «Réguler le bordel mondial : quand la féminisation de la migration continue, la prostitution devient le prototype de l'industrie mondiale, comment protégeons-nous ces travailleuses ${ }^{13} \gg$. Comment expliquer un tel engouement?

8. À quelques exceptions près. Voir, par exemple, Marlou Schrover, " Feminization and problematization of migration: Europe in the nineteenth and twentieth centuries ", in Dirk Hoerder, Amarjit Kaur (dir.), Proletarian and Gendered Mass Migrations: A Global Perspective on Continuities and Discontinuities from the 19th to the 21st Centuries, Leyde, Brill, 2013, pp. 103-131. 9. Hania Zlotnik, «International migration policies and the status of female migrants ", in International Migration Review, vol. 24, $n^{\circ}$ 2, 1990, pp. 372-381. 10. Nicola Piper, «Feminization of labor migration as violence against women: International, regional, and local nongovernmental organization responses in Asia ", in Violence Against Women, vol. 9, $\mathrm{n}^{\circ}$ 6, 2003, pp. 723745. 11. Zuhal Yesilyurt Gündüz, "The feminization of migration: Care and the new emotional imperialism ", Monthly Review, vol. $65, n^{\circ} 7,2013$, pp. 32-43. 12. Arun Kumar Acharya, "Feminization of migration and trafficking of women in Mexico ", in Revista de cercetare si interventie sociala, vol. 30, 2010, pp. 19-38. 13. Leah Platt, "Regulating the global brothel ", in The American Prospect, 2001, pp. S10-S14. 


\section{La théorie de la division internationale du travail}

L'étude de l'usage de l'expression « féminisation de la migration » montre un lien avec la critique néo-marxiste de la mondialisation. On affirme, par exemple, dans l'une des encyclopédies que "la féminisation de la migration a été mise en évidence depuis 1980 lors de la période de mondialisation économique rapide ${ }^{14}$ ". Ailleurs, on soutient que "les sciences sociales ont mis en évidence une croissance marquée du nombre de femmes migrantes, ainsi que de leur proportion dans le nombre total des migrants " et que " ce phénomène est lié à la transformation économique, politique, culturelle du capitalisme tardif's ». Les exemples sont nombreux.

S'il n'existe pas de féminisation de la migration dans les années 1980, on assiste dans cette période à l'émergence d'une théorie qui la prédit. C'est la théorie de la « restructuration économique mondiale " qui soutient qu'il existe une «nouvelle division internationale du travail » à laquelle la féminisation de la migration contribue. Cette théorie émerge à la fin des années 1970 comme une critique de la " mondialisation ". Dans un ouvrage intitulé La nouvelle division internationale du travail : le chômage structurel dans les pays industrialisés et l'industrialisation des pays en développement, Friedrich Fröbel et ses collègues tirent des conclusions sur la mondialisation à partir d'une enquête menée auprès des entreprises allemandes ${ }^{16}$. Ils affirment qu'une " restructuration économique mondiale " est en train de se produire par la délocalisation de certaines activités productives des pays de l'OCDE, où le chômage est estimé à $5 \%$, vers les pays en développe- ment. L'expansion du capital est supposée créer une "nouvelle division du travail » entre les pays anciennement colonisés qui s'industrialisent et les pays occidentaux qui se spécialisent dans les services. Les migrations Sud-Nord, caractérisées par l'expression de Marx " armée de réserve des travailleurs ", y sont expliquées comme le produit de l'expansion capitaliste.

Le lien entre l'expansion du capital et la migration des femmes est mieux articulé dans un livre sur la Mobilité du travail et du capital publié par Saskia Sassen en $1988^{17}$. Pour Sassen, il existe un lien causal entre l'investissement direct étranger et la migration des femmes. L'expansion du capital aboutit à produire une division internationale du travail entre, d'une part, des zones industrielles d'exportation des pays en développement et, d'autre part, des "villes mondiales" telles que New York et Los Angeles qui " contrôlent le système économique mondial ». Les deux types de lieux sont supposés attirer la migration des femmes vers les emplois peu qualifiés. D’un côté, les zones d'exportation "déracinent » les femmes du milieu rural et contri-
C'est la théorie de

la « restructuration économique mondiale» qui soutient qu'il existe une « nouvelle division internationale du travail » à laquelle la féminisation de la migration contribue. buent au "bouleversement des structures d'emploi traditionnel, souvent non-rémunéré ". Sassen insiste sur le fait que l'expansion capitaliste conduit au "recrutement massif de jeunes femmes [...], lesquelles ne seraient pas autrement entrées dans l'emploi rémunéré [un phénomène] dont on a montré qu'il contribue au chômage des hommes et, dans certains cas, à leur émigration ». D'un autre côté, les zones d'exportation préparent les femmes à l'émigration en les "occidentalisant ». La féminisation 


\section{REPÉRAGE}

de l'emploi serait suivie par la « pratique répandue du licenciement de nouveaux travailleurs, des jeunes femmes pour la plupart ", lesquelles « devenues sans emploi et occidentalisées n'ont d'autre choix que d'émigrer". Dans les villes mondiales, où la structure occupationnelle est supposée polarisée entre emplois hautement rémunérés et occupations mal payées, les immigrés vont "servir » les «styles de vie des professionnels à hauts revenus" (supposés être nonimmigrés). Pour Sassen, comme pour d'autres néo-marxistes, la migration n'est qu'un produit de l'expansion capitaliste dont les conséquences sont néfastes pour les travailleurs locaux : "l'immigration et la délocalisation sont des moyens de préserver une force de travail bon marché et de lutter contre les revendications des travailleurs organisés des pays développés ${ }^{18} "$.

La théorie de la division internationale du travail présuppose une chaîne causale complexe dans laquelle l'investissement direct étranger détermine le recrutement massif des femmes dans les pays du Sud (féminisation de l'emploi), lequel favorise leur migration vers les pays du Nord (féminisation de la migration), où les femmes vont occuper des emplois peu qualifiés, mal rémunérés ou genrés (division internationale et genrée du travail). Cette chaîne causale rend la théorie plausible, mais peu probable. La raison en est logique : la probabilité d'une conjonction des facteurs est plus faible que la probabilité de chacun de ces facteurs pris isolément. De fait, chaque chaînon de la théorie fait objet de controverses ou de contestations dans la littérature. La question de savoir si l'investissement direct étranger a un impact sur l'émigration et, dans l'affirmative, si cet impact est négatif ou positif est une question encore débattue aujourd'hui' ${ }^{19}$. La question de savoir si la migration interne est une condition nécessaire et suffisante de la migration internationale suscite des controverses ${ }^{20}$.

Malgré les controverses, l'idée d'une division internationale du travail est séduisante et bon nombre d'études tendent à l'illustrer. Elles se focalisent sur la présence des femmes originaires des pays en développement dans les emplois peu qualifiés - soit dans le secteur industriel du pays d'origine, soit dans le secteur des services du pays d'accueil. Le succès des recherches sur le care a amplifié l'intérêt pour les migrantes travaillant dans le service à la personne. Certaines analyses y voient une tendance mondiale théorisée comme "care drain²1", "mondialisation du maternage 22 " ou "impérialisme émotionnel23 ". La référence à la " féminisation de la migration » sert à illustrer la dimension genrée de la division du travail Nord-Sud.

\section{La féminisation de la migration qualifiée}

Les travaux évoquant la « féminisation de la migration » tendent à négliger les diplômes des femmes migrantes. Par exemple, Arlie Hochschild, créatrice du concept « care drain », considère que «la vie

Belonging in Contemporary Europe, New York/Oxford, Berghahn Books, 2010. 16. Friedrich Fröbel, Jürgen Heinrichs, Otto Kreye, The New International Division of Labour: Structural Unemployment in Industrialised Countries and Industrialisation in Developing Countries, Londres, Cambridge University Press, 1980. 17. Saskia Sassen, The Mobility of Labor and Capital: A Study in International Investment and Labor Flow, Cambridge, Cambridge University Press, 1988. 18. Saskia Sassen-Koob, " Notes on the incorporation of Third World women into wage-labor through immigration and off-shore production ", in International Migration Review, vol. 18, $n^{\circ}$ 4, 1984, pp. 1144-1167. 19. Pour une étude récente, voir Xu Xu, Kevin Sylwester, « The effects of foreign direct investment on emigration: The roles of FDI source country, education, and gender ", in Economic Modelling, vol. 55, 2016, pp. 401-409. 20. Nana Oishi, Women in Motion: Globalization, State Policies, and Labor Migration in Asia, Stanford, Stanford University Press, 2005. 21. Arlie Hochschild, "The nanny chain ", in American Prospect, vol. 11, $n^{\circ}$ 4, 2000, pp. 32-37. 22. Rhacel Salazar Parreñas, Servants of Globalization, Stanford, Stanford University Press, 2001. 23. Zuhal Yesilyurt Gündüz, "The feminization of migration: Care and the new emotional imperialism ", op. cit. 24. Arlie Russell Hochschild, "Le nouvel or du monde », in Nouvelles questions féministes, vol. 23, $n^{\circ}$ 3, 2004, pp. 59-74. 
de Rowena ", nounou philippine aux États-Unis, " reflète une tendance mondiale d'une ampleur croissante: l'importation de soins et d'amour des pays pauvres vers les pays riches ${ }^{24}$ ". Cependant, elle note que Rowena " a effectué des études d'ingénieure pendant trois ans " mais ne s'y attarde pas plus ${ }^{25}$. De la même manière, l'échantillon de travailleuses domestiques interviewées par Rhacel Parreñas contient pour moitié des femmes diplômées, mais son étude met en évidence la " mondialisation du maternage » et n'interroge pas le déclassement ou les ambitions professionnelles des migrantes ${ }^{26}$. Ses hypothèses sont façonnées par la théorie de la division internationale du travail que les femmes migrantes sont censées illustrer ${ }^{27}$.

Le désintérêt de la théorie de la division internationale du travail pour les diplômes des femmes migrantes conduit à obscurcir un phénomène important. Depuis 1980, on assiste à une tendance importante à la " féminisation de la migration qualifiée » vers les pays riches, et ce dans le sens statistique le plus exigeant que l'on peut donner à cette expression.

En effet, une signification alternative de la « féminisation de la migration " a été récemment

\section{Tableau 2. Composition de stocks de migrants (25 ans+) par sexe, origine et niveau d'études dans 20 pays de l'OCDE (1980 et 2010)}

\begin{tabular}{|c|c|c|c|c|}
\hline & \multicolumn{2}{|c|}{$\begin{array}{l}\text { FEMMES PARMI } \\
\text { LES MIGRANTS DIPLOMÉS } \\
\text { DE L'ENSEIGNEMENT } \\
\text { SUPERIEUR }\end{array}$} & \multicolumn{2}{|c|}{$\begin{array}{l}\text { DIPLOMÉES } \\
\text { DE L'ENSEIGNEMENT } \\
\text { SUPERIEUR PARMIS } \\
\text { LES FEMMES MIGRANTES }\end{array}$} \\
\hline & 1980 & 2010 & 1980 & 2010 \\
\hline TOUTES ORIGINES & $45 \%$ & $51 \%$ & $18 \%$ & $40 \%$ \\
\hline \multicolumn{5}{|l|}{ PAR NIVEAU DE REVENU } \\
\hline Pays développés & $47 \%$ & $52 \%$ & $17 \%$ & $45 \%$ \\
\hline Pays en développement & $41 \%$ & $51 \%$ & $20 \%$ & $38 \%$ \\
\hline Dont pays les moins avancés & $38 \%$ & $45 \%$ & $23 \%$ & $36 \%$ \\
\hline \multicolumn{5}{|l|}{ PAR RÉGION } \\
\hline Afrique & $34 \%$ & $44 \%$ & $15 \%$ & $38 \%$ \\
\hline Afrique Subsaharienne & $34 \%$ & $46 \%$ & $24 \%$ & $45 \%$ \\
\hline Asie & $42 \%$ & $51 \%$ & $26 \%$ & $51 \%$ \\
\hline Europe de l'Est & $42 \%$ & $53 \%$ & $20 \%$ & $41 \%$ \\
\hline Europe & $46 \%$ & $51 \%$ & $14 \%$ & $39 \%$ \\
\hline Pays membres de I'UE & $46 \%$ & $51 \%$ & $14 \%$ & $39 \%$ \\
\hline Amérique & $48 \%$ & $54 \%$ & $23 \%$ & $33 \%$ \\
\hline Amérique latine et Caraïbes & $47 \%$ & $53 \%$ & $19 \%$ & $30 \%$ \\
\hline Océanie & $47 \%$ & $55 \%$ & $28 \%$ & $55 \%$ \\
\hline
\end{tabular}

Source : Base de données Brücker et al. et calculs de l'auteure.

25. Speranta Dumitru, "Care drain : le piège sexiste du nationalisme », in Tiziana Caponio, Fedora Giordano, Luisa Ricaldone (dir.), World Wide Women. Globalizzazione, generi, linguaggio, Turin, CIRSDe/University of Turin, 2011, pp. 51-60 ; Speranta Dumitru, «From “brain drain” to "care drain”: Women's labor migration and methodological sexism ", in Women's Studies International Forum, vol. 47, $\mathrm{n}^{\circ}$ 2, 2014, pp. 203-212. 26. Rhacel Salazar Parreñas, Servants of Globalization, op. cit. Les caractéristiques de l'échantillon sont décrites dans l'annexe. 27. Rhacel Salazar Parreñas, " Migrant Filipina domestic workers and the international division of reproductive labor ", in Gender and Society, vol. 14, n 4, 2000, pp. 560-580. 28. Katharine M. Donato, Donna Gabaccia, Gender and International Migration, New York, Russell Sage Foundation, 2015. 


\section{REPÉRAGE}

proposée par Katharine Donato et Donna Gabac$\mathrm{cia}^{28}$. Jugeant excessive l'habitude de réserver "l'équilibre entre les sexes " à la seule proportion égale d'hommes et de femmes, elles proposent de considérer l'intervalle $47-53 \%$ comme équilibré, en le distinguant d'une composition prédominante masculine (53-75\%) ou à forte prédominance masculine (au-delà de $75 \%$ ). La "féminisation de la migration " serait alors un changement d'une composition à (forte) prédominance masculine vers une composition hommes/femmes équilibrée.

Cette typologie nous permet de faire remarquer que, depuis les années 1980, on assiste à une féminisation non pas de la migration tout court mais de la migration qualifiée. Sur un échantillon de 20 pays de l'OCDE, les données montrent que la proportion des femmes parmi les migrants diplômés du supérieur, toutes origines confondues, augmente de $45 \%$ en 1980 à $51 \%$ en 2010 (cf. tableau 2). En trente ans, la migration qualifiée est passée d'une composition prédominante masculine à une composition équilibrée entre les sexes. Ce changement est visible pour l'émigration originaire aussi bien des pays développés que des pays en développement. Le constat est plus nuancé par région géographique, la migration qualifiée en provenance de certaines régions étant équilibrée depuis 1980 (Amérique, Océanie), devenant en 2010 prédominant-féminine (Europe de l'Est, Amé-

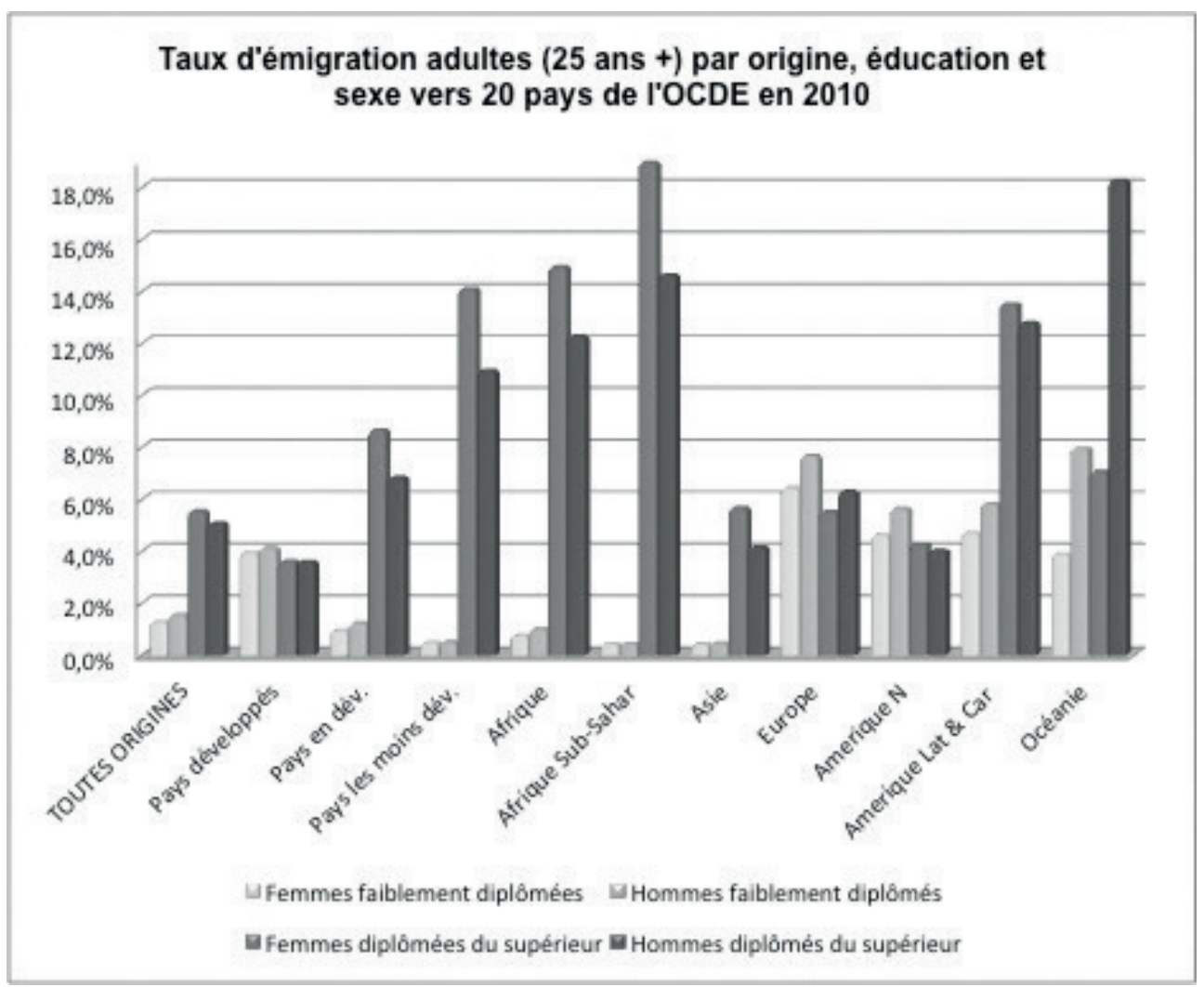

Source : Base de données Brücker et al. et calcul de l'auteure. 
rique) ou étant encore prédominant-masculine (Afrique). Tout comme la proportion des femmes dans la migration qualifiée, la part des diplômées parmi les femmes migrantes a augmenté quelle que soit l'origine considérée.

Plus important, le diplôme fournit aux femmes un véritable passeport : le taux d'émigration des femmes diplômées de l'enseignement supérieur, toutes origines confondues, est quatre fois plus élevé que celui des autres femmes (voir graphique 1). Ce décalage augmente de façon considérable pour les femmes originaires des pays en développement et à faible revenu. Pour les femmes originaires de l'Afrique subsaharienne, ce décalage est 44 fois plus élevé que le taux d'émigration des femmes moins diplômées qui n'excède pas $0,4 \%$.

\section{Conclusion}

Pour les femmes originaires des pays en développement, l'absence de diplôme constitue un frein à la mobilité internationale. Les politiques d'immigration sélective expliquent sans doute pourquoi le diplôme constitue une source d'inégalité de mobilité entre les femmes, mais elles n'expliquent pas pourquoi cette inégalité affecte beaucoup plus les femmes nées dans les pays pauvres. Les études inspirées par la théorie de la division internationale du travail négligent cette réalité statistique. Elles associent la féminisation de la migration aux emplois peu qualifiés sans envisager le déclassement professionnel, un phénomène qui affecte les femmes nées dans les pays en développement. 